\title{
Novel Chaos Secure Communication System Based on Walsh Code
}

\author{
Gang Zhang, Niting Cui, and Tianqi Zhang \\ School of Communication and Information Engineering, Chongqing University of Posts and Telecommunications, \\ Chongqing 400065, China
}

Correspondence should be addressed to Niting Cui; 1276707796@qq.com

Received 26 March 2015; Revised 15 June 2015; Accepted 2 July 2015

Academic Editor: George S. Tombras

Copyright (c) 2015 Gang Zhang et al. This is an open access article distributed under the Creative Commons Attribution License, which permits unrestricted use, distribution, and reproduction in any medium, provided the original work is properly cited.

A multiuser communication scheme which is a hybrid of Walsh code with DCSK and CDSK is proposed to improve low data transmission rate of Differential Chaos Shift Keying (DCSK), poor bit error ratio (BER) performance of Correlation Delay Shift Keying (CDSK), and disadvantage of orthogonality in traditional multiuser DCSK. It not only overcomes the disadvantages of DCSK and CDSK, but also has better performance than CDSK and higher transmission data rate than DCSK. It has been proved that the novel multiuser CDSK-DCSK has better properties than traditional Multiple Input Multiple Output-Differential Chaos Shift Keying (MIMO-DCSK) and Modified-Differential Chaos Shift Keying (M-DCSK). Also the multiuser interference is greatly suppressed due to the orthogonality of Walsh code.

\section{Introduction}

Spread spectrum technology is used in communication system to bear low data rate information by using spread spectrum sequence due to its broad bandwidth characteristics. The technology has advantages such as high security, anti-interference, and antimultipath fading and being easy to be realized in code division multiple access (CDMA) [1]. In recent years, chaotic spread spectrum communication system has been deeply researched in spread spectrum technology [2]. It is different from the traditional spread spectrum technology since the carriers are high speed chaotic signals which are generated by different chaotic maps. Chaotic signal has the advantages of high bandwidth, being nonperiodic, being difficult to predict, and good autocorrelation and cross correlation features [3].

According to the way of demodulation, chaotic communication system is divided into two types: the coherent and the noncoherent demodulation [4]. Coherent demodulation needs the receiver to reconstruct chaotic signal, which means that the security and noise immunity are better than noncoherent demodulation. But it is difficult to realize chaotic synchronization. The security in noncoherent demodulation is worse than in coherent demodulation, but it is easy to implement and the cost of hardware is much lower. In the existing noncoherent systems, reference signal is used for dispreading in most of the receivers [5-10]. Differential Chaos Shift Keying (DCSK) has disadvantages of low data rate [11]. In Correlation Delay Shift Keying (CDSK), the data rate is 2 times that of DCSK [12], but the BER performance is worse than DCSK [13]. Much attention has been attracted since the concept of multiple access DCSK has been proposed [14]. In [15], different interval between transmit signal and carrier is used to distinguish different users. But the orthogonality is poor when using the smaller spread factor.

A combination of multiuser CDSK-DCSK with Walsh coded scheme is proposed in this paper. It can not only overcome the disadvantages of DCSK and CDSK, which means that the BER is better than CDSK and the data rate is higher than DCSK, but also suppress multiuser interference well, due to the orthogonality of Walsh code.

\section{Novel Multiuser CDSK-DCSK System}

The novel multiuser CDSK-DCSK scheme is shown in Figure 1, where the system has $U$ users totally, and the $u$ th user is discussed for special purpose. 


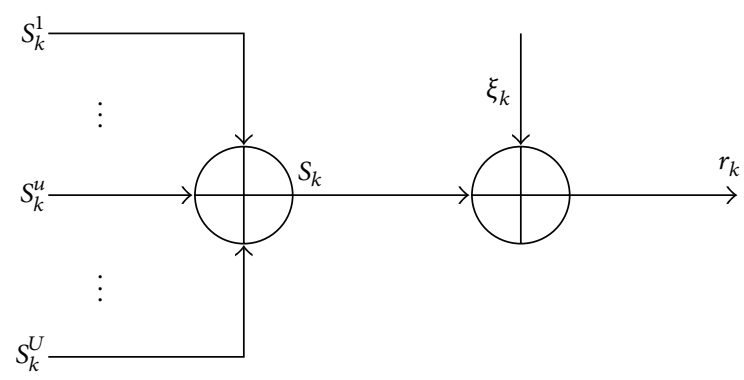

FIgURE 1: $U$ users through Additive White Gaussian Noise (AWGN) channel.

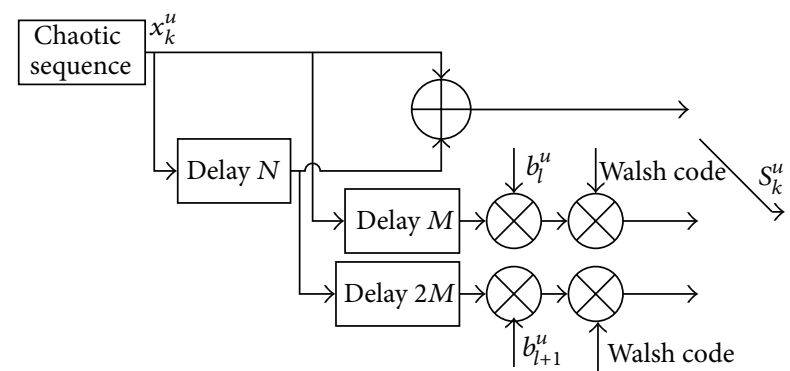

FIgure 2: Transmitting end of user $u$.

Chaotic signal is generated by the logistic map firstly. Then, chaotic sequence $x_{k}$ is generated after the symbolic function mapping as follows:

$$
\begin{aligned}
y_{k+1} & =1-2 y_{k}^{2}, \quad y_{k} \in(-1,1), \\
x_{k}^{u} & =\operatorname{sgn}\left(y_{k}\right),
\end{aligned}
$$

$$
k=0,1,2, \ldots,
$$

where $y_{k}$ is chaotic signal, $\operatorname{sgn}()$ is symbolic function, and $x_{k}^{u}$ is chaotic sequence of the $u$ th user.

The transmitter is illustrated in Figure 2. A pair of bit $\left(b_{l}^{u}, b_{l+1}^{u}\right)$ is modulated and transmitted in a frame, where $b_{l}^{u}, b_{l+1}^{u} \in\{+1,-1\}$. In the $l$ th frame, $x_{k}^{u}$ and $x_{k-N}^{u}$ are transmitted in the first slot, where $N$ is the time delay of $x_{k}^{u}$ and $N>M$. In the second and third slots, $b_{l}^{u}$ and $b_{l+1}^{u}$ are multiplied with chaotic sequence and the assigned Walsh code, respectively. Transmitting signal of $u$ th user is shown in

$$
\begin{aligned}
& s_{k}^{u} \\
& = \begin{cases}x_{k}^{u}+x_{k-N}^{u}, & 3 l M+1<k<(3 l+1) M, \\
b_{l}^{u} x_{k-M}^{u} w_{1, k}^{u}, & (3 l+1) M+1<k<(3 l+2) M, \\
b_{l+1}^{u} x_{k-N-2 M}^{u} w_{2, k}^{u}, & (3 l+2) M+1<k<(3 l+3) M,\end{cases}
\end{aligned}
$$

where $M$ is the spread factor. $w_{1, k}^{u}$ and $w_{2, k}^{u}$ are Walsh code of $u$ th user.

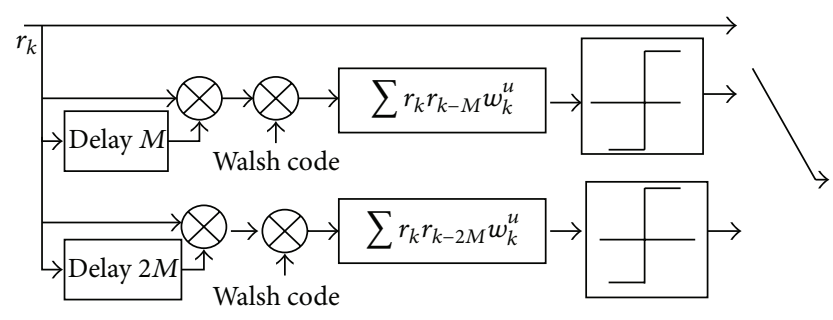

FIGURE 3: Receiving end of user $u$.

From Figure 1 and (2), the total transmitting signal $s_{k}$ is easy to be obtained. The received signal after transmission is shown as follows:

$$
\begin{aligned}
r_{k} & =s_{k}+\xi_{k}=\sum_{u=1}^{U} s_{k}^{u}+\xi_{k} \\
& = \begin{cases}\sum_{u=1}^{U}\left(x_{k}^{u}+x_{k-N}^{u}\right)+\xi_{k}, & 3 l M+1<k<(3 l+1) M, \\
\sum_{u=1}^{U}\left(b_{l}^{u} x_{k-M}^{u} w_{1, k}^{u}\right)+\xi_{k}, & (3 l+1) M+1<k<(3 l+2) M, \\
\sum_{u=1}^{U}\left(b_{l+1}^{u} x_{k-N-2 M}^{u} w_{2, k}^{u}\right)+\xi_{k}, & (3 l+2) M+1<k<(3 l+3) M,\end{cases}
\end{aligned}
$$

where $\xi_{k}$ is assumed as Additive White Gaussian Noise (AWGN) and $U$ is the total number of users.

Figure 3 shows the receiver's structure. Walsh code is multiplied with $r_{k}$ and $r_{k-M}$ or $r_{k-2 M}$. The Walsh code in the $u$ th receiver must agree with the one of the $u$ th transmitter. After correlation demodulation the original signal is obtained and is shown in

$$
\begin{aligned}
c_{l}^{u}= & \sum_{k=1}^{M} r_{k} r_{k-M} w_{1, k}^{u} \\
= & \sum_{k=1}^{M}\left(\left(s_{k}+\xi_{k}\right)\left(s_{k-M}+\xi_{k-M}\right) w_{1, k}^{u}\right) \\
= & \sum_{i=1}^{U} \sum_{j=1}^{U} \sum_{k=1}^{M} b_{l}^{j} x_{k-M}^{i} x_{k-M}^{j} w_{1, k}^{j} w_{1, k}^{u}+\sum_{i=1}^{U} \sum_{k=1}^{M} x_{k-M}^{i} \xi_{k} w_{1, k}^{u} \\
& +\sum_{i=1}^{U} \sum_{k=1}^{M} x_{k-N-M}^{i} \xi_{k} w_{1, k}^{u} \\
& +\sum_{j=1}^{U} \sum_{k=1}^{M} b_{l}^{j} x_{k-M}^{j} w_{1, k}^{j} w_{1, k}^{u} \xi_{k-M} \\
& +\sum_{i=1}^{U} \sum_{j=1}^{U} \sum_{k=1}^{M} b_{l}^{j} x_{k-M}^{j} x_{k-N-M}^{i} w_{1, k}^{j} w_{1, k}^{u} \\
& +\sum_{k=1}^{M} \xi_{k} \xi_{k-M} w_{1, k}^{u} .
\end{aligned}
$$


In order to simplify the output, $c_{l}^{u}$ can be divided into three parts $A, B$, and $C$ as follows:

$$
\begin{aligned}
A= & \sum_{i=1}^{U} \sum_{j=1}^{U} \sum_{k=1}^{M} b_{l}^{j} x_{k-M}^{i} x_{k-M}^{j} w_{1, k}^{j} w_{1, k}^{u} \\
= & \sum_{i=1 ; i \neq j}^{U} \sum_{j=1}^{U}\left(\sum_{k=1}^{M} b_{l}^{j} x_{k-M}^{i} x_{k-M}^{j} w_{1, k}^{j} w_{1, k}^{u}\right) \\
& +\sum_{j=1 ; j \neq u}^{U}\left(\sum_{k=1}^{M} b_{l}^{j} x_{k-M}^{j} x_{k-M}^{j} w_{1, k}^{j} w_{1, k}^{u}\right)+b_{l}^{u} M, \\
B= & \sum_{i=1}^{U} \sum_{k=1}^{M} x_{k-M}^{i} \xi_{k} w_{1, k}^{u}+\sum_{i=1}^{U} \sum_{k=1}^{M} x_{k-N-M}^{i} \xi_{k} w_{1, k}^{u} \\
& +\sum_{j=1}^{U} \sum_{k=1}^{M} b_{l}^{j} x_{k-M}^{j} w_{1, k}^{j} \xi_{k-M} w_{1, k}^{u} \\
& +\sum_{i=1}^{U} \sum_{j=1}^{U} \sum_{k=1}^{M} b_{l}^{j} x_{k-M}^{j} w_{1, k}^{j} x_{k-N-M}^{i} w_{1, k}^{u}, \\
C= & \sum_{k=1}^{M} \xi_{k} \xi_{k-M} w_{1, k}^{u} .
\end{aligned}
$$

The chaotic sequence has the following properties [16]:

(1) Chaotic sequence generated by the same map but with different initial value is noncorrelated.

(2) The chaotic sequence is the same as impulse function after normalized autocorrelation.

Besides, due to the orthogonal property of Walsh code such as $\sum_{k=1}^{M} w_{k}^{j} w_{k}^{u}=0, w_{k}^{u} w_{k}^{u}=1$, and $x_{k}^{u} x_{k}^{u}=1, b_{l}^{u} M$ in (5) is the only useful signal and the rest of (5), (6), and (7) are interference. The first item is the cross correlation of chaotic sequence and the second item equals $0 . b_{l}^{u}$ can be demodulated according to the following rules:

$$
b_{l}^{u}=f\left(c_{l}^{u}\right)= \begin{cases}-1, & c_{l}^{u}<0, \\ +1, & c_{l}^{u} \geq 0 .\end{cases}
$$

Similarly, the decision rules for $b_{l+1}^{u}$ are

$$
b_{l+1}^{u}=f\left(c_{l+1}^{u}\right)= \begin{cases}-1, & c_{l+1}^{u}<0, \\ +1, & c_{l+1}^{u} \geq 0 .\end{cases}
$$

\section{Performance Analysis}

By central limit theorem, the correlation output approximately obeys the normal distribution. The mean and variance of $c_{l}^{u}$ are required to get the system's BER. Features of chaotic sequence and Walsh code are presented in [17].

(1) For different chaotic sequences $x_{i}(i=0,1,2, \ldots)$ and $x_{j}(j=0,1,2, \ldots)$ generated by the same map, $E\left[x_{i} x_{j}\right]=E\left[x_{i}\right] E\left[x_{j}\right]=0$ and $\operatorname{var}\left[x_{i} x_{j}\right]=$ $\operatorname{var}\left[x_{i}\right] \operatorname{var}\left[x_{j}\right]=1$, when $i \neq j$.
(2) For different Walsh codes $w_{k}^{p}$ and $w_{k}^{q}$, where $p, q \in$ $[1, U]$ and $k=(1,2, \ldots, M)$, when $p \neq q$, $\operatorname{var}\left[w_{k}^{p} w_{k}^{q}\right]=\operatorname{var}\left[w_{k}^{p}\right]=\operatorname{var}\left[w_{k}^{q}\right]=1$.

(3) Correlation among chaotic sequences, AWGN and Walsh codes, is 0 .

(4) For (4), $\operatorname{cov}[A, B]=\operatorname{cov}[A, C]=\operatorname{cov}[B, C]=0$.

Suppose the $u$ th user's first bit in $l$ th frame is " +1 ":

$$
E\left\{c_{l}^{u} \mid b_{l}^{u}=+1\right\}=E A+E B+E C=M,
$$

where $E$ represents the mean of $c_{l}^{u}$. Consider

$$
\begin{aligned}
\operatorname{var}( & A)=\operatorname{var}\left(\sum_{i=1 ; i \neq j}^{U} \sum_{j=1}^{U} \sum_{k=1}^{M} x_{k-M}^{i} x_{k-M}^{j} w_{1, k}^{j} w_{1, k}^{u}+M\right) \\
= & \sum_{i=1 ; i \neq j}^{U} \sum_{j=1}^{U} \sum_{k=1}^{M} \operatorname{var}\left(x_{k-M}^{i} x_{k-M}^{j}\right) \operatorname{var}\left(w_{1, k}^{j} w_{1, k}^{u}\right) \\
= & M(U-1)^{2} \\
\operatorname{var}( & B) \\
= & \sum_{i=1}^{U} \sum_{k=1}^{M} \operatorname{var}\left(x_{k-M}^{i} \xi_{k} w_{1, k}^{u}\right) \\
& +\sum_{i=1}^{U} \sum_{k=1}^{M} \operatorname{var}\left(x_{k-N-M}^{i} \xi_{k} w_{1, k}^{u}\right) \\
& +\sum_{j=1}^{U} \sum_{k=1}^{M} \operatorname{var}\left(x_{k-M}^{j} w_{1, k}^{j} \xi_{k-M} w_{1, k}^{u}\right) \\
& +\sum_{i=1}^{U} \sum_{j=1}^{U} \sum_{k=1}^{M} \operatorname{var}\left(x_{k-M}^{j} w_{1, k}^{j} x_{k-N-M}^{i} w_{1, k}^{u}\right) \\
= & \sum_{i=1}^{U} \sum_{k=1}^{M} \operatorname{var}\left(x_{k-M}^{i}\right) \operatorname{var}\left(\xi_{k}\right) \operatorname{var}\left(w_{1, k}^{u}\right) \\
& +\sum_{i=1}^{U} \sum_{k=1}^{M} \operatorname{var}\left(x_{k-N-M}^{i}\right) \operatorname{var}\left(\xi_{k}\right) \operatorname{var}\left(w_{1, k}^{u}\right) \\
& +\sum_{j=1}^{U} \sum_{k=1}^{M} \operatorname{var}\left(x_{k-M}^{j}\right) \operatorname{var}\left(\xi_{k-M}\right) \operatorname{var}\left(w_{1, k}^{u}\right) \\
& \sum_{k=1}^{U} \sum_{k}, \\
& \\
& \\
&
\end{aligned}
$$

where $D$ represents variance and $N_{0} / 2$ is noise power density. 
So the variance of $c_{l}^{u}$ is

$$
\begin{aligned}
& D\left\{c_{l}^{u} \mid b_{l}^{u}=+1\right\} \\
&= D[A]+D[B]+D[C] \\
&+2\{\operatorname{cov}[A, B]+\operatorname{cov}[A, C]+\operatorname{cov}[B, C]\} \\
&=M\left(U^{2}-U\right)+2 M U N_{0}+\frac{1}{4} M N_{0}^{2},
\end{aligned}
$$

where cov represents covariance.

Similarly, when $b_{l}^{u}=-1$, the mean and variance of $c_{l}^{u}$ are

$$
\begin{aligned}
& E\left\{c_{l}^{u} \mid b_{l}^{u}=-1\right\}=-M \\
& D\left\{c_{l}^{u} \mid b_{l}^{u}=-1\right\}=M\left(U^{2}-U\right)+2 M U N_{0}+\frac{1}{4} M N_{0}^{2}
\end{aligned}
$$

The system's bit error ratio (BER) is

$$
\begin{aligned}
& \mathrm{BER}=\frac{1}{2} P\left(c_{l}^{u}>0 \mid b_{l}^{u}=-1\right)+\frac{1}{2} P\left(c_{l}^{u}<0 \mid b_{l}^{u}=+1\right) \\
& =\frac{1}{2} \operatorname{erfc}\left(\frac{E\left(c_{l}^{u} \mid b_{l}^{u}=+1\right)}{\sqrt{2 D\left(c_{l}^{u} \mid b_{l}^{u}=+1\right)}}\right)=\frac{1}{2} \\
& \cdot \operatorname{erfc} \frac{M}{\sqrt{2 M(U-1)^{2}+4 M U N_{0}+(1 / 2) M N_{0}^{2}}}=\frac{1}{2} \\
& \cdot \operatorname{erfc}\left(\frac{2(U-1)^{2}}{M}+12 U\left(\frac{E_{b}}{N_{0}}\right)^{-1}\right. \\
& \left.+\frac{9 M}{2}\left(\frac{E_{b}}{N_{0}}\right)^{-2}\right)^{-1 / 2},
\end{aligned}
$$

where erfc is the error function, $\operatorname{erfc}(\varphi)=(2 /$ $\sqrt{\pi}) \int_{\varphi}^{\infty} \exp \left(-x^{2}\right) d x$, and $E_{b}$ is bit energy, $E_{b}=3 M \operatorname{var}\left[x_{i}\right]$.

From (14), with a certain value of $U$ and $E_{b} / N_{o}$, there exists $M_{\text {opt }}$ to realize the best system performance. Suppose $y=2(U-1)^{2} / M+12 U\left(E_{b} / N_{0}\right)^{-1}+(9 M / 2)\left(E_{b} / N_{0}\right)^{-2}$. It is easy to obtain $M_{\text {opt }}$ after differentiating $y$ :

$$
y^{\prime}=-\frac{2(U-1)^{2}}{M^{2}}+\frac{9}{2}\left(\frac{E_{b}}{N_{0}}\right)^{-2} .
$$

Suppose that $y^{\prime}=0$; the equation of $M_{\mathrm{opt}}$ is as follows:

$$
M_{\mathrm{opt}}=\frac{2(U-1)}{3} \frac{E_{b}}{N_{0}} .
$$

By (16), for certain $E_{b} / N_{o}$, under different $U, M_{\mathrm{opt}}$ is different. For example, suppose $E_{b} / N_{0}=10 \mathrm{~dB}$; when $U=3$ and $U=5, M_{\text {opt }}$ is 13.33 and 26.66 , respectively.

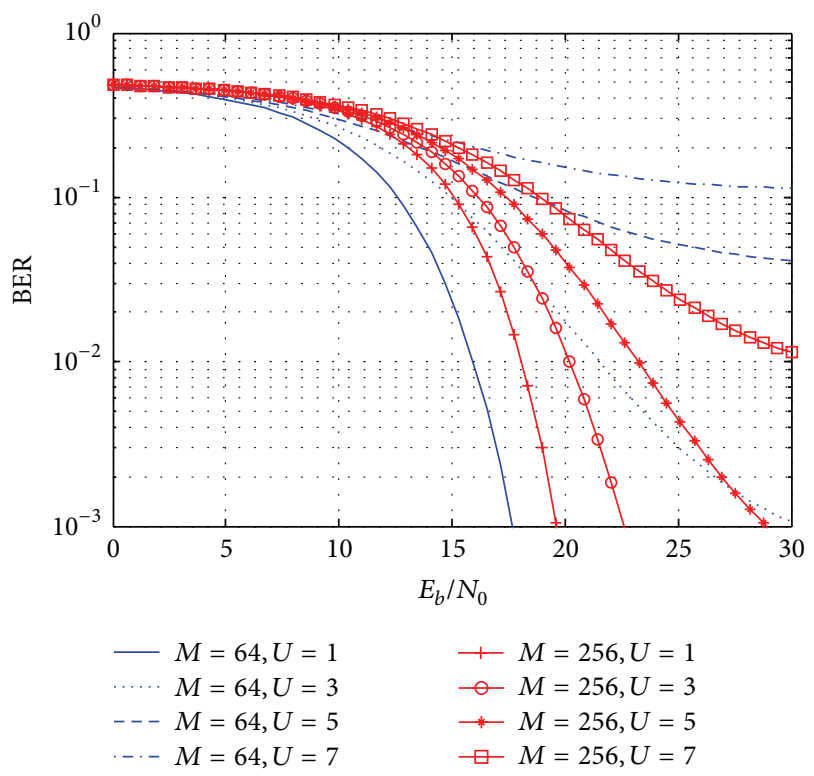

FIGURE 4: BER performance versus $E_{b} / N_{0}$ for different spreading factor $M$.

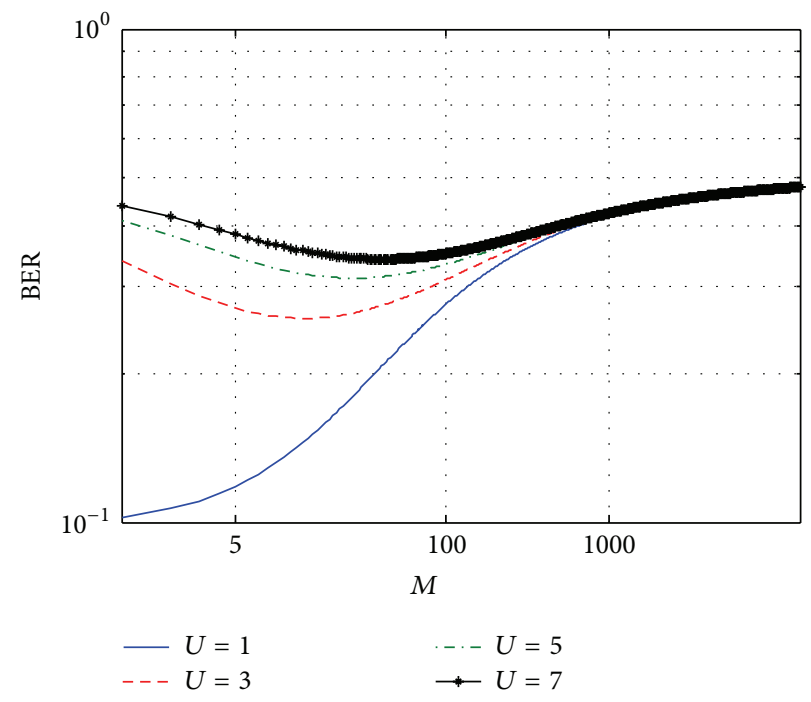

FIGURE 5: BER performance versus spreading factor $M$ under $E_{b} / N_{0}=10 \mathrm{~dB}$.

\section{Simulation Comparisons}

Figure 4 shows that the smaller the value of $M$, the stronger the influence on BER by $E_{b} / N_{0}$. It is obvious that the intervals between different curves under the same $E_{b} / N_{0}$ in $M=64$ are significantly larger than that of $M=256$. With $M$ increasing, BER gets smaller and the system's performance gets better.

Figure 5 shows that selecting an appropriate $M$ has great impact on the system's performance. On one hand, there exists an optimum $M$ to achieve the best BER. If $M$ is increased continuously, the system's performance gets worse. On the other hand, the transmission efficiency is too low if $M$ is too large. 


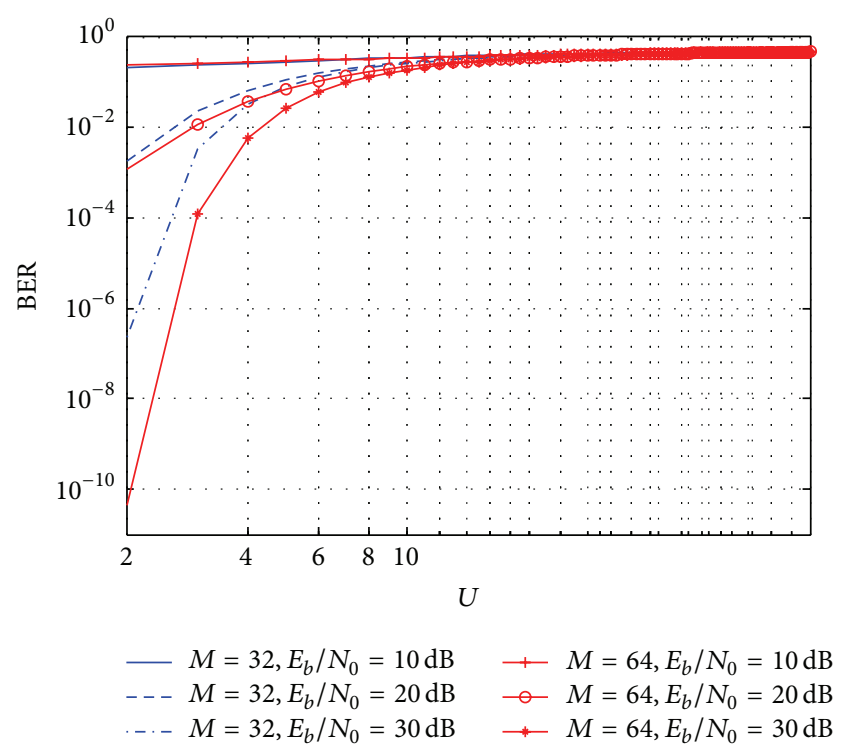

FIGURE 6: BER performance versus different total number of users.

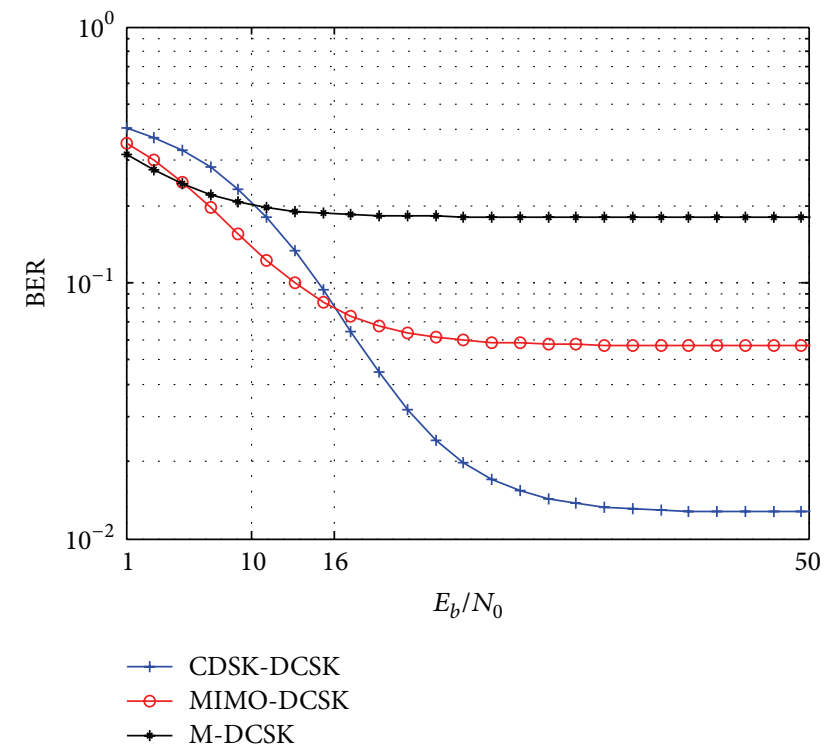

Figure 7: Simulated BERs versus $E_{b} / N_{0}$ for multiuser CDSK-DCSK, MIMO-DCSK, and M-DCSK with $M=5$ and $U=2$.

Figure 6 displays that multiuser interference increases with the users' total number increasing, so the system's performance gets worse. Under different $E_{b} / N_{0}$, the BER gradually tends to be constant and unrelated with $M$.

It can be seen from Figure 7 that the performance of multiuser CDSK-DCSK is slightly inferior to MIMO-DCSK [11] when $E_{b} / N_{0}$ is low. But the proposed system's data rate is 2 times that of MIMO-DCSK. When $E_{b} / N_{0}>16 \mathrm{~dB}$, the proposed system's performance is much better than that of MIMO-DCSK. Compared with M-DCSK proposed in [9], in low $E_{b} / N_{0}$, there is not much difference between multiuser CDSK-DCSK and M-DCSK. But when $E_{b} / N_{0}>10 \mathrm{~dB}$, the proposed system's BER is improved one-order magnitude.

\section{Conclusion}

The excellent autocorrelation and cross correlation characteristics of chaotic signal are used in traditional multiuser DCSK system [15] to distinguish different users. When the spread factor is small, the orthogonality between different chaotic signals is poor. A hybrid CDSK-DCSK combined with Walsh code system is proposed in this paper to realize the multiuser transmission. The signal is transmitted in pairs, so transmitting rate is 2 times the traditional ones. Interference between different users can be reduced due to the application of Walsh code. Also, the simulation results show that, under the same circumstances, the performance of multiuser CDSK-DCSK system is much better than that of M-DCSK and MIMO-DCSK, especially when $E_{b} / N_{0}$ is larger than $16 \mathrm{~dB}$ and $10 \mathrm{~dB}$, respectively.

\section{Conflict of Interests}

The authors declare that there is no conflict of interests regarding the publication of this paper.

\section{Acknowledgments}

This research is supported by the National Natural Science Foundation of China (Grants nos. 61371164, 61071196, and 61102131), the Program for New Century Excellent Talents in University (Grant no. NCET-10-0927), the Project of Key Laboratory of Signal and Information Processing of Chongqing (Grant no. CSTC2009CA2003), the Chongqing Distinguished Youth Foundation (Grant no. CSTC2011jjiq40002), the Natural Science Foundation of Chongqing (Grants nos. CSTC2010BB2398, CSTC2010BB2409, CSTC2010BB2411, and CSTC2012JJA40008), and the Research Project of Chongqing Educational Commission (Grants KJ120525 and KJ130524).

\section{References}

[1] G. Kolumban, M. P. Kennedy, Z. Jako, and G. Kis, "Chaotic communications with correlator receivers: theory and performance limits," Proceedings of the IEEE, vol. 90, no. 5, pp. 711-732, 2002.

[2] G. Kolumban, M. P. Kennedy, and L. O. Chua, "The role of synchronization in digital communications using chaos. II. Chaotic modulation and chaotic synchronization," IEEE Transactions on Circuits and Systems. I. Fundamental Theory and Applications, vol. 45, no. 11, pp. 1129-1140, 1998.

[3] L. F. He, G. Zhang, and T. Q. Zhang, "A secure image transmission scheme based on improved DCSK," Telecommunications Science, no. 6, pp. 94-99, 2013.

[4] Z. G. Chen and W. K. Xu, "Performance analysis of CSDCSK over Nakagami-m fading channels," Journal of Chongqing University of Posts and Telecommunications, vol. 24, pp. 395-399, 2012.

[5] H. Yang and G.-P. Jiang, "Reference-modulated DCSK: a novel chaotic communication scheme," IEEE Transactions on Circuits and Systems II: Express Briefs, vol. 60, no. 4, pp. 232-236, 2013.

[6] G. Kolumban, Z. Jako, and M. P. Kennedy, "Enhanced versions of DCSK and FM-DCSK data transmission systems," in Proceedings of the IEEE International Symposium on Circuits and 
Systems (ISCAS '99), vol. 4, pp. 475-478, Orlando, Fla, USA, 1999.

[7] J.-Y. Duan, G.-P. Jiang, and H. Yang, "A new chaotic communication scheme: differential correlation delay shift Keying," in Proceedings of the International Conference on Communications, Circuits and Systems (ICCCAS '13), pp. 446-449, IEEE, Chengdu, China, November 2013.

[8] W. N. Tam, F. C. M. Lau, and C. K. Tse, "Generalized correlationdelay-shift-keying scheme for noncoherent chaos-based communication systems," IEEE Transactions on Circuits and Systems I: Regular Papers, vol. 53, no. 3, pp. 712-721, 2006.

[9] H. Yang and G.-P. Jiang, "High-efficiency differential-chaosshift-keying scheme for chaos-based noncoherent communication," IEEE Transactions on Circuits and Systems II: Express Briefs, vol. 59, no. 5, pp. 312-316, 2012.

[10] G. Kaddoum and F. Gagnon, "Design of a high-data-rate differential chaos-shift keying system," IEEE Transactions on Circuits and Systems II: Express Briefs, vol. 59, no. 7, pp. 448452, 2012.

[11] S. P. Li, L. Wang, and Y. L. Xia, "System devising and simulation of FM-DCSK in non-flat fading channel," Journal of Chongqing University of Posts and Telecommunications, vol. 17, pp. 282-286, 2005.

[12] M. Sushchik, L. S. Tsimring, and A. R. Volkovskii, "Performance analysis of correlation-based communication schemes utilizing chaos," IEEE Transactions on Circuits and Systems I: Fundamental Theory and Applications, vol. 47, no. 12, pp. 1684-1691, 2000.

[13] Q. Ding and J. N. Wang, "Design of frequency-modulated correlation delay shift keying chaotic communication system," IET Communications, vol. 5, no. 7, pp. 901-905, 2011.

[14] M. P. Kennedy, G. Kolumban, G. Kis, and Z. Jako, "Recent advances in communicating with chaos," in Proceedings of the IEEE International Symposium on Circuits and Systems (ISCAS '98), vol. 4, pp. 461-464, IEEE, Monterey, Calif, USA, May-June 1998.

[15] F. C. M. Lau, M. M. Yip, C. K. Tse, and S. F. Hau, "A multiple-access technique for differential chaos-shift keying," IEEE Transactions on Circuits and Systems I: Fundamental Theory and Applications, vol. 49, no. 1, pp. 96-104, 2002.

[16] S. Mandal and S. Banerjee, "Analysis and CMOS implementation of a chaos-based communication system," IEEE Transactions on Circuits and Systems I: Regular Papers, vol. 51, no. 9, pp. 1708-1722, 2004.

[17] Z.-B. Zhou, T. Zhou, and J.-X. Wang, "Performance analysis of an improved multiple-access differential chaos shift keying," Journal of Xidian University, vol. 36, no. 4, pp. 730-735, 2009. 

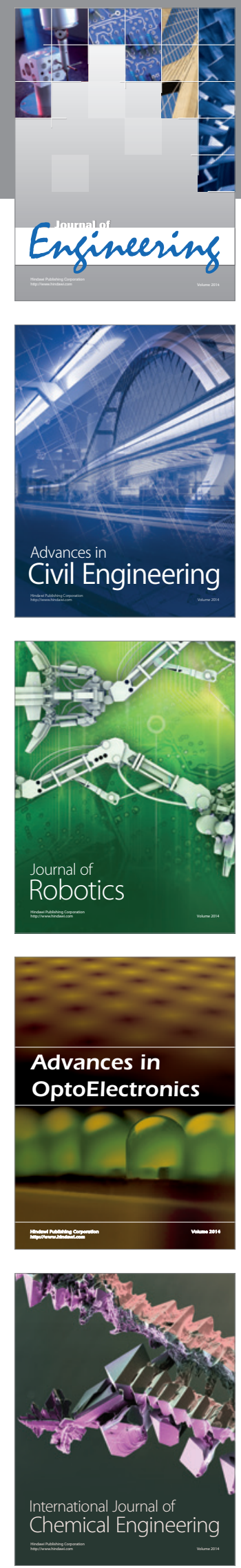

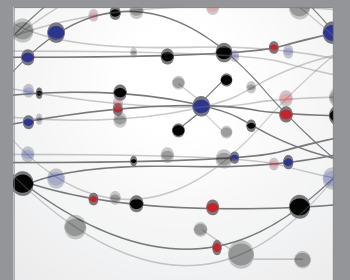

The Scientific World Journal
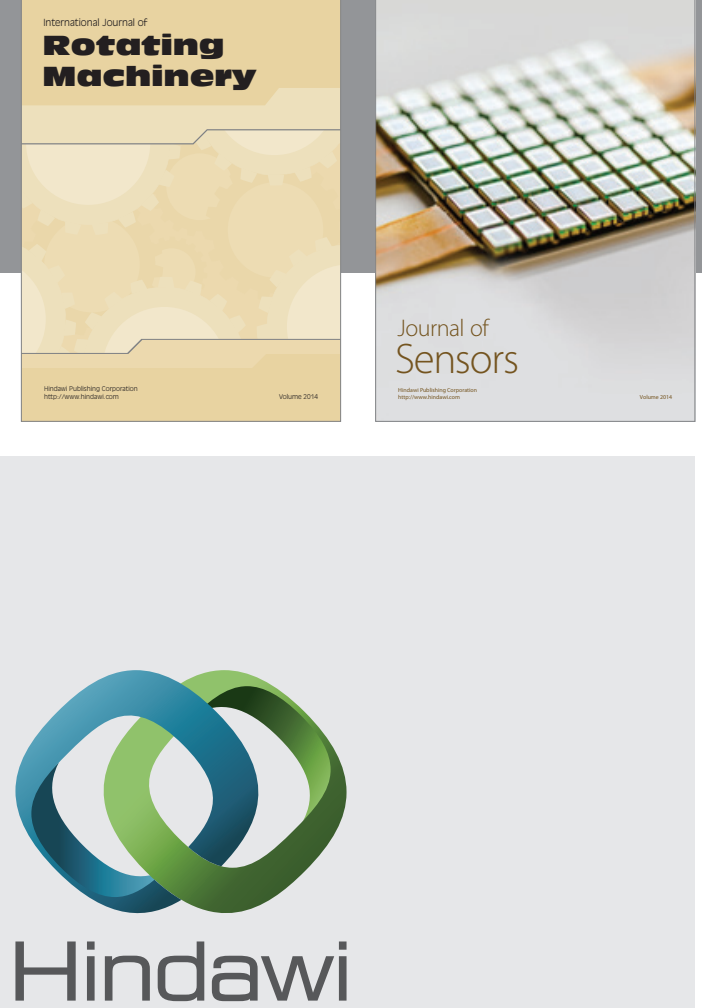

Submit your manuscripts at http://www.hindawi.com
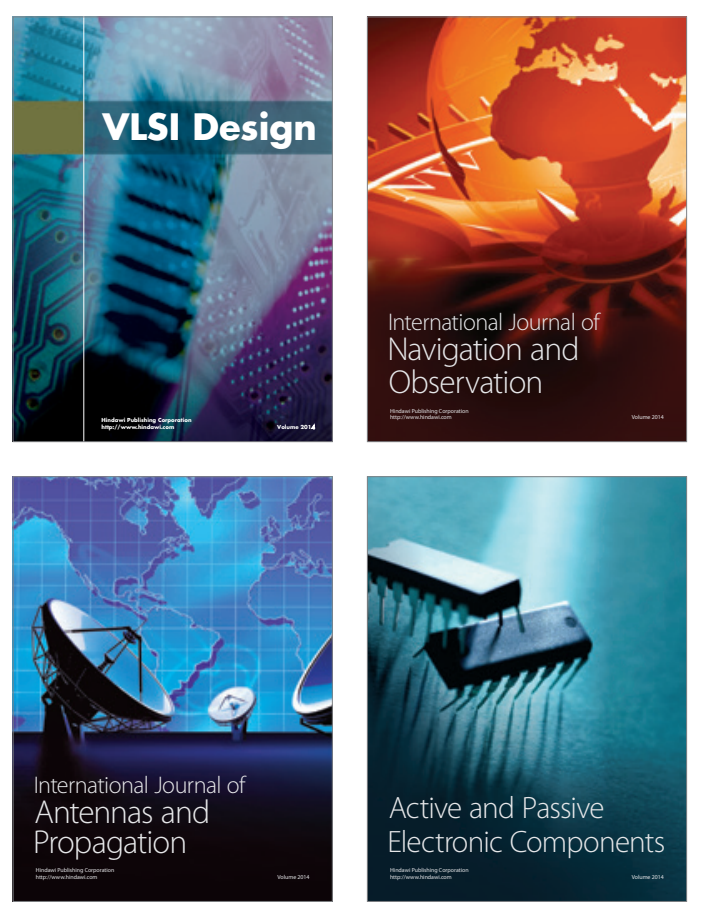
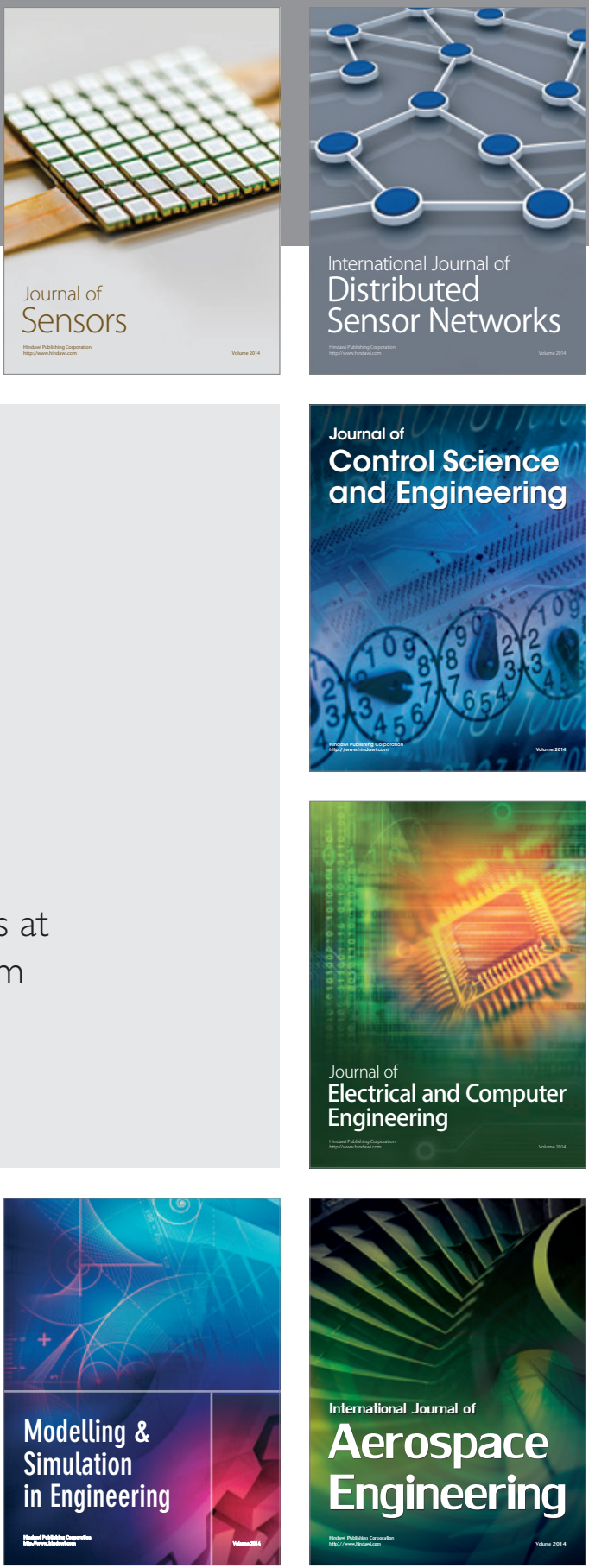

Journal of

Control Science

and Engineering
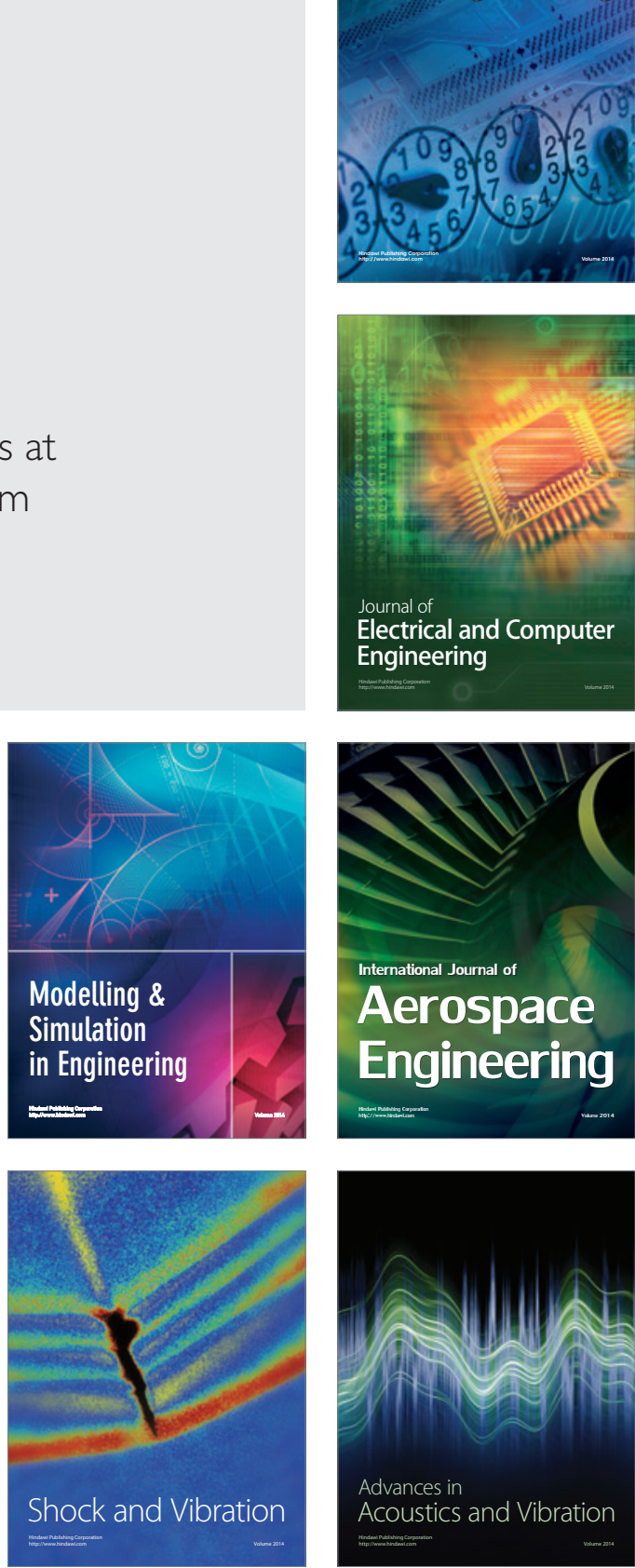